CUBO A Mathematical Journal

Vol.21, № 02, (01-13). August 2019

\title{
Caputo fractional Iyengar type Inequalities
}

\author{
George A. Anastassiou \\ Department of Mathematical Sciences, \\ University of Memphis, Memphis, TN 38152, U.S.A. \\ ganastss@memphis.edu
}

\begin{abstract}
Here we present Caputo fractional Iyengar type inequalities with respect to $\mathrm{L}_{p}$ norms, with $1 \leq p \leq \infty$. The method is based on the right and left Caputo fractional Taylor's formulae.

\section{RESUMEN}

Aquí presentamos desigualdades de tipo Caputo fraccional Iyengar con respecto a las normas $\mathrm{L}_{p}$, con $1 \leq p \leq \infty$. El método se basa en las fórmulas de Taylor fraccionales de Caputo derecha e izquierda.
\end{abstract}

Keywords and Phrases: Iyengar inequality, right and left Caputo fractional, Taylor formulae, Caputo fractional derivative.

2010 AMS Mathematics Subject Classification: 26A33, 26D10, 26D15. 


\section{Introduction}

We are motivated by the following famous Iyengar inequality (1938), 4].

Theorem 1. Let $\mathrm{f}$ be a differentiable function on $[\mathrm{a}, \mathrm{b}]$ and $\left|\mathrm{f}^{\prime}(\mathrm{x})\right| \leq \mathrm{M}$. Then

$$
\left|\int_{a}^{b} f(x) d x-\frac{1}{2}(b-a)(f(a)+f(b))\right| \leq \frac{M(b-a)^{2}}{4}-\frac{(f(b)-f(a))^{2}}{4 M}
$$

We need

Definition 2. ([1], p. 394) Let $v>0, n=\lceil v\rceil(\lceil\cdot\rceil$ the ceiling of the number $), f \in A C^{n}([a, b])$ (i.e. $\mathrm{f}^{(\mathrm{n}-1)}$ is absolutely continuous on $[\mathrm{a}, \mathrm{b}]$ ). The left Caputo fractional derivative of order $v$ is defined as

$$
D_{* a}^{v} f(x)=\frac{1}{\Gamma(n-v)} \int_{a}^{x}(x-t)^{n-v-1} f^{(n)}(t) d t
$$

$\forall x \in[a, b]$, and it exists almost everywhere over $[\mathrm{a}, \mathrm{b}]$.

We need

Definition 3. ([2], p. 336-337) Let $v>0, n=\lceil v\rceil, f \in A C^{n}([a, b])$. The right Caputo fractional derivative of order $v$ is defined as

$$
D_{b-}^{v} f(x)=\frac{(-1)^{n}}{\Gamma(n-v)} \int_{x}^{b}(z-x)^{n-v-1} f^{(n)}(z) d z
$$

$\forall x \in[a, b]$, and exists almost everywhere over $[\mathrm{a}, \mathrm{b}]$.

\section{Main Results}

We present the following Caputo fractional Iyengar type inequalities:

Theorem 4. Let $v>0, \mathrm{n}=\lceil\mathrm{v}\rceil\left(\lceil\cdot\rceil\right.$ is the ceiling of the number), and $\mathrm{f} \in \mathrm{AC}^{\mathrm{n}}([\mathrm{a}, \mathrm{b}])$ (i.e. $\mathrm{f}^{(\mathrm{n}-1)}$ is absolutely continuous on $\left.[\mathrm{a}, \mathrm{b}]\right)$. We assume that $\mathrm{D}_{* \mathrm{a}}^{v} \mathrm{f}, \mathrm{D}_{\mathrm{b}-}^{v} \mathrm{f} \in \mathrm{L}_{\infty}([\mathrm{a}, \mathrm{b}])$. Then

i)

$$
\begin{gathered}
\left|\int_{a}^{b} f(x) d x-\sum_{k=0}^{n-1} \frac{1}{(k+1) !}\left[f^{(k)}(a)(t-a)^{k+1}+(-1)^{k} f^{(k)}(b)(b-t)^{k+1}\right]\right| \leq \\
\quad \frac{\max \left\{\left\|D_{* a}^{v} f\right\|_{L_{\infty}([a, b])},\left\|D_{b-}^{v} f\right\|_{L_{\infty}([a, b])}\right\}}{\Gamma(v+2)}\left[(t-a)^{v+1}+(b-t)^{v+1}\right],
\end{gathered}
$$

$\forall \mathrm{t} \in[\mathrm{a}, \mathrm{b}]$, 
ii) at $\mathrm{t}=\frac{\mathrm{a}+\mathrm{b}}{2}$, the right hand side of (4) is minimized, and we get:

$$
\begin{gathered}
\left|\int_{a}^{b} f(x) d x-\sum_{k=0}^{n-1} \frac{1}{(k+1) !} \frac{(b-a)^{k+1}}{2^{k+1}}\left[f^{(k)}(a)+(-1)^{k} f^{(k)}(b)\right]\right| \leq \\
\frac{\max \left\{\left\|D_{* a}^{v} f\right\|_{L_{\infty}([a, b])},\left\|D_{b-}^{v} f\right\|_{L_{\infty}([a, b])}\right\}}{\Gamma(v+2)} \frac{(b-a)^{v+1}}{2^{v}},
\end{gathered}
$$

iii) if $\mathrm{f}^{(\mathrm{k})}(\mathrm{a})=\mathrm{f}^{(\mathrm{k})}(\mathrm{b})=0$, for all $\mathrm{k}=0,1, \ldots, \mathrm{n}-1$, we obtain

$$
\left|\int_{a}^{b} f(x) d x\right| \leq \frac{\max \left\{\left\|D_{* a}^{v} f\right\|_{L_{\infty}([a, b])},\left\|D_{b-}^{v} f\right\|_{L_{\infty}([a, b])}\right\}}{\Gamma(v+2)} \frac{(b-a)^{v+1}}{2^{v}}
$$

which is a sharp inequality,

iv) more generally, for $\mathbf{j}=0,1,2, \ldots, \mathbf{N} \in \mathbb{N}$, it holds

$$
\begin{aligned}
& \left|\int_{a}^{b} f(x) d x-\sum_{k=0}^{n-1} \frac{1}{(k+1) !}\left(\frac{b-a}{N}\right)^{k+1}\left[j^{k+1} f^{(k)}(a)+(-1)^{k}(N-j)^{k+1} f^{(k)}(b)\right]\right| \\
& \quad \leq \frac{\max \left\{\left\|D_{* a}^{v} f\right\|_{L_{\infty}([a, b])},\left\|D_{b-}^{v} f\right\|_{L_{\infty}([a, b])}\right\}}{\Gamma(v+2)}\left(\frac{b-a}{N}\right)^{v+1}\left[j^{v+1}+(N-j)^{v+1}\right],
\end{aligned}
$$

v) if $\mathrm{f}^{(\mathrm{k})}(\mathrm{a})=\mathrm{f}^{(\mathrm{k})}(\mathrm{b})=0, \mathrm{k}=1, \ldots, \mathrm{n}-1$, from (7) we get:

$$
\begin{gathered}
\left|\int_{a}^{b} f(x) d x-\left(\frac{b-a}{N}\right)[j f(a)+(N-j) f(b)]\right| \leq \\
\frac{\max \left\{\left\|D_{* a}^{v} f\right\|_{L_{\infty}([a, b])},\left\|D_{b-}^{v} f\right\|_{L_{\infty}([a, b])}\right\}}{\Gamma(v+2)}\left(\frac{b-a}{N}\right)^{v+1}\left[j^{v+1}+(N-j)^{v+1}\right],
\end{gathered}
$$

$j=0,1,2, \ldots, N$,

vi) when $\mathrm{N}=2$ and $\mathrm{j}=1$, (8) turns to

$$
\begin{gathered}
\left|\int_{a}^{b} f(x) d x-\left(\frac{b-a}{2}\right)(f(a)+f(b))\right| \leq \\
\frac{\max \left\{\left\|D_{* a}^{v} f\right\|_{L_{\infty}([a, b])},\left\|D_{b-}^{v} f\right\|_{L_{\infty}([a, b])}\right\}}{\Gamma(v+2)} \frac{(b-a)^{v+1}}{2^{v}},
\end{gathered}
$$

vii) when $0<v \leq 1$, inequality (9) is again valid without any boundary conditions. 
Proof. Let $v>0, n=\lceil v\rceil$, and $f \in A C^{n}([a, b])$. Then by ([3], p. 54) left Caputo fractional Taylor's formula we have

$$
f(x)-\sum_{k=0}^{n-1} \frac{f^{(k)}(a)}{k !}(x-a)^{k}=\frac{1}{\Gamma(v)} \int_{a}^{x}(x-t)^{v-1} D_{* a}^{v} f(t) d t,
$$

$\forall x \in[\mathrm{a}, \mathrm{b}]$.

Also by ([2], p. 341) right Caputo fractional Taylor's formula we get:

$$
f(x)-\sum_{k=0}^{n-1} \frac{f^{(k)}(b)}{k !}(x-b)^{k}=\frac{1}{\Gamma(v)} \int_{x}^{b}(z-x)^{v-1} D_{b-}^{v} f(z) d z,
$$

$\forall x \in[\mathrm{a}, \mathrm{b}]$.

By (10) we derive

$$
\left|f(x)-\sum_{k=0}^{n-1} \frac{f^{(k)}(a)}{k !}(x-a)^{k}\right| \leq \frac{\left\|D_{* a}^{v} f\right\|_{L_{\infty}([a, b])}}{\Gamma(v+1)}(x-a)^{v},
$$

and by (11) we obtain

$$
\left|f(x)-\sum_{k=0}^{n-1} \frac{f^{(k)}(b)}{k !}(x-b)^{k}\right| \leq \frac{\left\|D_{b-}^{v} f\right\|_{L_{\infty}([a, b])}}{\Gamma(v+1)}(b-x)^{v},
$$

$\forall x \in[a, b]$.

Call

$$
\gamma_{1}:=\frac{\left\|D_{* a}^{v} f\right\|_{L_{\infty}([a, b])}}{\Gamma(v+1)}
$$

and

$$
\gamma_{2}:=\frac{\left\|D_{b-}^{v} f\right\|_{L_{\infty}([a, b])}}{\Gamma(v+1)} .
$$

Set

$$
\gamma:=\max \left(\gamma_{1}, \gamma_{2}\right)
$$

That is

$$
\left|f(x)-\sum_{k=0}^{n-1} \frac{f^{(k)}(a)}{k !}(x-a)^{k}\right| \leq \gamma(x-a)^{v},
$$

and

$$
\left|f(x)-\sum_{k=0}^{n-1} \frac{f^{(k)}(b)}{k !}(x-b)^{k}\right| \leq \gamma(b-x)^{v},
$$

$\forall x \in[\mathrm{a}, \mathrm{b}]$. 
Hence it holds

$$
\sum_{k=0}^{n-1} \frac{f^{(k)}(a)}{k !}(x-a)^{k}-\gamma(x-a)^{v} \leq f(x) \leq \sum_{k=0}^{n-1} \frac{f^{(k)}(a)}{k !}(x-a)^{k}+\gamma(x-a)^{v}
$$

and

$$
\sum_{k=0}^{n-1} \frac{f^{(k)}(b)}{k !}(x-b)^{k}-\gamma(b-x)^{v} \leq f(x) \leq \sum_{k=0}^{n-1} \frac{f^{(k)}(b)}{k !}(x-b)^{k}+\gamma(b-x)^{v},
$$

$\forall x \in[a, b]$.

Let any $t \in[a, b]$, then by integration over $[a, t]$ and $[t, b]$, respectively, we obtain

$$
\begin{gathered}
\sum_{k=0}^{n-1} \frac{f^{(k)}(a)}{(k+1) !}(t-a)^{k+1}-\frac{\gamma}{(v+1)}(t-a)^{v+1} \leq \int_{a}^{t} f(x) d x \leq \\
\sum_{k=0}^{n-1} \frac{f^{(k)}(a)}{(k+1) !}(t-a)^{k+1}+\frac{\gamma}{(v+1)}(t-a)^{v+1},
\end{gathered}
$$

and

$$
\begin{gathered}
-\sum_{k=0}^{n-1} \frac{f^{(k)}(b)}{(k+1) !}(t-b)^{k+1}-\frac{\gamma}{(v+1)}(b-t)^{v+1} \leq \int_{t}^{b} f(x) d x \leq \\
-\sum_{k=0}^{n-1} \frac{f^{(k)}(b)}{(k+1) !}(t-b)^{k+1}+\frac{\gamma}{(v+1)}(b-t)^{v+1} .
\end{gathered}
$$

Adding (21) and (22), we obtain

$$
\begin{gathered}
\left\{\sum_{k=0}^{n-1} \frac{1}{(k+1) !}\left[f^{(k)}(a)(t-a)^{k+1}-f^{(k)}(b)(t-b)^{k+1}\right]\right\}- \\
\frac{\gamma}{(v+1)}\left[(t-a)^{v+1}+(b-t)^{v+1}\right] \leq \int_{a}^{b} f(x) d x \leq \\
\left\{\sum_{k=0}^{n-1} \frac{1}{(k+1) !}\left[f^{(k)}(a)(t-a)^{k+1}-f^{(k)}(b)(t-b)^{k+1}\right]\right\}+ \\
\frac{\gamma}{(v+1)}\left[(t-a)^{v+1}+(b-t)^{v+1}\right],
\end{gathered}
$$

$\forall \mathrm{t} \in[\mathrm{a}, \mathrm{b}]$.

Consequently we derive:

$$
\begin{gathered}
\left|\int_{a}^{b} f(x) d x-\sum_{k=0}^{n-1} \frac{1}{(k+1) !}\left[f^{(k)}(a)(t-a)^{k+1}+(-1)^{k} f^{(k)}(b)(b-t)^{k+1}\right]\right| \leq \\
\frac{\gamma}{(v+1)}\left[(t-a)^{v+1}+(b-t)^{v+1}\right]
\end{gathered}
$$


$\forall \mathrm{t} \in[\mathrm{a}, \mathrm{b}]$.

Let us consider

$$
g(t):=(t-a)^{v+1}+(b-t)^{v+1}, \forall t \in[a, b] .
$$

Hence

$$
g^{\prime}(t)=(v+1)\left[(t-a)^{v}-(b-t)^{v}\right]=0,
$$

giving $(t-a)^{v}=(b-t)^{v}$ and $t-a=b-t$, that is $t=\frac{a+b}{2}$ the only critical number here.

We have $g(a)=g(b)=(b-a)^{v+1}$, and $g\left(\frac{a+b}{2}\right)=\frac{(b-a)^{v+1}}{2^{v}}$, which the minimum of $g$ over $[a, b]$.

Consequently the right hand side of (24) is minimized when $t=\frac{a+b}{2}$, with value $\frac{\gamma}{(v+1)} \frac{(b-a)^{v+1}}{2^{v}}$.

Assuming $f^{(k)}(a)=f^{(k)}(b)=0$, for $k=0,1, \ldots, n-1$, then we obtain that

$$
\left|\int_{a}^{b} f(x) d x\right| \leq \frac{\gamma}{(v+1)} \frac{(b-a)^{v+1}}{2^{v}}
$$

which is a sharp inequality.

When $t=\frac{a+b}{2}$, then (24) becomes

$$
\begin{gathered}
\left|\int_{a}^{b} f(x) d x-\sum_{k=0}^{n-1} \frac{1}{(k+1) !} \frac{(b-a)^{k+1}}{2^{k+1}}\left[f^{(k)}(a)+(-1)^{k} f^{(k)}(b)\right]\right| \leq \\
\frac{\gamma}{(v+1)} \frac{(b-a)^{v+1}}{2^{v}} .
\end{gathered}
$$

Next let $N \in \mathbb{N}, j=0,1,2, \ldots, N$ and $t_{j}=a+j\left(\frac{b-a}{N}\right)$, that is $t_{0}=a, t_{1}=a+\frac{b-a}{N}, \ldots, t_{N}=b$.

Hence it holds

$$
t_{j}-a=j\left(\frac{b-a}{N}\right), \quad\left(b-t_{j}\right)=(N-j)\left(\frac{b-a}{N}\right), j=0,1,2, \ldots, N .
$$

We notice that

$$
\left(t_{j}-a\right)^{v+1}+\left(b-t_{j}\right)^{v+1}=\left(\frac{b-a}{N}\right)^{v+1}\left[j^{v+1}+(N-j)^{v+1}\right]
$$

$j=0,1,2, \ldots, N$,

and (for $k=0,1, \ldots, n-1)$

$$
\begin{gathered}
{\left[f^{(k)}(a)\left(t_{j}-a\right)^{k+1}+(-1)^{k} f^{(k)}(b)\left(b-t_{j}\right)^{k+1}\right]=} \\
{\left[f^{(k)}(a) j^{k+1}\left(\frac{b-a}{N}\right)^{k+1}+(-1)^{k} f^{(k)}(b)(N-j)^{k+1}\left(\frac{b-a}{N}\right)^{k+1}\right]=}
\end{gathered}
$$




$$
\left(\frac{b-a}{N}\right)^{k+1}\left[f^{(k)}(a) j^{k+1}+(-1)^{k} f^{(k)}(b)(N-j)^{k+1}\right]
$$

$j=0,1,2, \ldots, N$.

By (24) we get

$$
\begin{gathered}
\left|\int_{a}^{b} f(x) d x-\sum_{k=0}^{n-1} \frac{1}{(k+1) !}\left(\frac{b-a}{N}\right)^{k+1}\left[f^{(k)}(a) j^{k+1}+(-1)^{k} f^{(k)}(b)(N-j)^{k+1}\right]\right| \\
\leq \frac{\gamma}{(v+1)}\left(\frac{b-a}{N}\right)^{v+1}\left[j^{v+1}+(N-j)^{v+1}\right],
\end{gathered}
$$

$j=0,1,2, \ldots, N$.

If $f^{(k)}(a)=f^{(k)}(b)=0, k=1, \ldots, n-1$, then (30) becomes

$$
\begin{gathered}
\left|\int_{a}^{b} f(x) d x-\left(\frac{b-a}{N}\right)[j f(a)+(N-j) f(b)]\right| \leq \\
\frac{\gamma}{(v+1)}\left(\frac{b-a}{N}\right)^{v+1}\left[j^{v+1}+(N-j)^{v+1}\right],
\end{gathered}
$$

$j=0,1,2, \ldots, N$.

When $N=2$ and $j=1$, then (31) becomes

$$
\begin{aligned}
& \left|\int_{a}^{b} f(x) d x-\left(\frac{b-a}{2}\right)(f(a)+f(b))\right| \leq \\
& \frac{\gamma}{(v+1)} 2\left(\frac{b-a}{2}\right)^{v+1}=\frac{\gamma}{(v+1)} \frac{(b-a)^{v+1}}{2^{v}} .
\end{aligned}
$$

Let $0<v \leq 1$, then $n=\lceil v\rceil=1$. In that case, without any boundary conditions, we derive from (32) again that

$$
\left|\int_{a}^{b} f(x) d x-\left(\frac{b-a}{2}\right)(f(a)+f(b))\right| \leq \frac{\gamma}{(v+1)} \frac{(b-a)^{v+1}}{2^{v}} .
$$

The theorem is proved in all cases.

We give

Theorem 5. Let $v \geq 1, n=\lceil v\rceil$, and $f \in A C^{n}([a, b])$. We assume that $D_{* a}^{v} f, D_{b-}^{v} f \in L_{1}([a, b])$. Then

i)

$$
\left|\int_{a}^{b} f(x) d x-\sum_{k=0}^{n-1} \frac{1}{(k+1) !}\left[f^{(k)}(a)(t-a)^{k+1}+(-1)^{k} f^{(k)}(b)(b-t)^{k+1}\right]\right| \leq
$$




$$
\frac{\max \left\{\left\|D_{* a}^{v} f\right\|_{L_{1}([a, b])},\left\|D_{b-}^{v} f\right\|_{L_{1}([a, b])}\right\}}{\Gamma(v+1)}\left[(t-a)^{v}+(b-t)^{v}\right],
$$

$\forall \mathrm{t} \in[\mathrm{a}, \mathrm{b}]$,

ii) when $v=1$, from (34), we have

$$
\begin{gathered}
\left|\int_{a}^{b} f(x) d x-[f(a)(t-a)+f(b)(b-t)]\right| \leq \\
\left\|f^{\prime}\right\|_{L_{1}([a, b])}(b-a), \quad \forall t \in[a, b],
\end{gathered}
$$

iii) from (35), we obtain ( $\mathrm{v}=1$ case)

$$
\left|\int_{a}^{b} f(x) d x-\left(\frac{b-a}{2}\right)(f(a)+f(b))\right| \leq\left\|f^{\prime}\right\|_{L_{1}([a, b])}(b-a),
$$

iv) at $\mathrm{t}=\frac{\mathrm{a}+\mathrm{b}}{2}, v>1$, the right hand side of (34) is minimized, and we get:

$$
\begin{gathered}
\left|\int_{a}^{b} f(x) d x-\sum_{k=0}^{n-1} \frac{1}{(k+1) !} \frac{(b-a)^{k+1}}{2^{k+1}}\left[f^{(k)}(a)+(-1)^{k} f^{(k)}(b)\right]\right| \leq \\
\frac{\max \left\{\left\|D_{* a}^{v} f\right\|_{L_{1}([a, b])},\left\|D_{b-}^{v} f\right\|_{L_{1}([a, b])}\right\}}{\Gamma(v+1)} \frac{(b-a)^{v}}{2^{v-1}},
\end{gathered}
$$

v) if $\mathrm{f}^{(\mathrm{k})}(\mathrm{a})=\mathrm{f}^{(\mathrm{k})}(\mathrm{b})=0$, for all $\mathrm{k}=0,1, \ldots, \mathrm{n}-1 ; \mathrm{v}>1$, from (37), we obtain

$$
\left|\int_{a}^{b} f(x) d x\right| \leq \frac{\max \left\{\left\|D_{* a}^{v} f\right\|_{L_{1}([a, b])},\left\|D_{b-}^{v} f\right\|_{L_{1}([a, b])}\right\}}{\Gamma(v+1)} \frac{(b-a)^{v}}{2^{v-1}}
$$

which is a sharp inequality,

vi) more generally, for $\mathbf{j}=0,1,2, \ldots, \mathrm{N} \in \mathbb{N}$, it holds

$$
\begin{gathered}
\left|\int_{a}^{b} f(x) d x-\sum_{k=0}^{n-1} \frac{1}{(k+1) !}\left(\frac{b-a}{N}\right)^{k+1}\left[j^{k+1} f^{(k)}(a)+(-1)^{k}(N-j)^{k+1} f^{(k)}(b)\right]\right| \\
\leq \frac{\max \left\{\left\|D_{* a}^{v} f\right\|_{L_{1}([a, b])},\left\|D_{b-}^{v} f\right\|_{L_{1}([a, b])}\right\}}{\Gamma(v+1)}\left(\frac{b-a}{N}\right)^{v}\left[j^{v}+(N-j)^{v}\right],
\end{gathered}
$$

vii) if $\mathrm{f}^{(\mathrm{k})}(\mathrm{a})=\mathrm{f}^{(\mathrm{k})}(\mathrm{b})=0, \mathrm{k}=1, \ldots, \mathrm{n}-1$, from (39) we get:

$$
\left|\int_{a}^{b} f(x) d x-\left(\frac{b-a}{N}\right)[j f(a)+(N-j) f(b)]\right| \leq
$$




$$
\frac{\max \left\{\left\|D_{* a}^{v} f\right\|_{L_{1}([a, b])},\left\|D_{b-}^{v} f\right\|_{L_{1}([a, b])}\right\}}{\Gamma(v+1)}\left(\frac{b-a}{N}\right)^{v}\left[j^{v}+(N-j)^{v}\right],
$$

$j=0,1,2, \ldots, N$,

viii) when $\mathrm{N}=2$ and $\mathrm{j}=1$, (40) turns to

$$
\begin{gathered}
\left|\int_{a}^{b} f(x) d x-\frac{(b-a)}{2}(f(a)+f(b))\right| \leq \\
\frac{\max \left\{\left\|D_{* a}^{v} f\right\|_{L_{1}([a, b])},\left\|D_{b-}^{v} f\right\|_{L_{1}([a, b])}\right\}}{\Gamma(v+1)} \frac{(b-a)^{v}}{2^{v-1}} .
\end{gathered}
$$

Proof. Here $v \geq 1$ and $D_{* a}^{v} f, D_{b-}^{v} f \in L_{1}([a, b])$. By (10) we get

$$
\begin{gathered}
\left|f(x)-\sum_{k=0}^{n-1} \frac{f^{(k)}(a)}{k !}(x-a)^{k}\right| \leq \frac{1}{\Gamma(v)}(x-a)^{v-1} \int_{a}^{x}\left|D_{* a}^{v} f(t)\right| d t \\
\leq \frac{(x-a)^{v-1}}{\Gamma(v)}\left\|D_{* a}^{v} f\right\|_{L_{1}([a, b])}
\end{gathered}
$$

$\forall x \in[a, b]$.

That is

$$
\left|f(x)-\sum_{k=0}^{n-1} \frac{f^{(k)}(a)}{k !}(x-a)^{k}\right| \leq \frac{\left\|D_{* a}^{v} f\right\|_{L_{1}([a, b])}}{\Gamma(v)}(x-a)^{v-1},
$$

$\forall x \in[\mathrm{a}, \mathrm{b}]$.

By (11) we get

$$
\begin{gathered}
\left|f(x)-\sum_{k=0}^{n-1} \frac{f^{(k)}(b)}{k !}(x-b)^{k}\right| \leq \frac{1}{\Gamma(v)}(b-x)^{v-1} \int_{x}^{b}\left|D_{b-}^{v} f(z)\right| d z \\
\leq \frac{(b-x)^{v-1}}{\Gamma(v)}\left\|D_{b-}^{v} f\right\|_{L_{1}([a, b])},
\end{gathered}
$$

$\forall x \in[\mathrm{a}, \mathrm{b}]$.

That is

$$
\left|f(x)-\sum_{k=0}^{n-1} \frac{f^{(k)}(b)}{k !}(x-b)^{k}\right| \leq \frac{\left\|D_{b-}^{v} f\right\|_{L_{1}([a, b])}}{\Gamma(v)}(b-x)^{v-1},
$$

$\forall x \in[a, b]$.

Call

$$
\delta_{1}:=\frac{\left\|D_{* a}^{v} f\right\|_{L_{1}([a, b])}}{\Gamma(v)}
$$


and

$$
\delta_{2}:=\frac{\left\|D_{b-}^{v} f\right\|_{L_{1}([a, b])}}{\Gamma(v)}
$$

Set

$$
\delta:=\max \left(\delta_{1}, \delta_{2}\right)
$$

That is

$$
\left|f(x)-\sum_{k=0}^{n-1} \frac{f^{(k)}(a)}{k !}(x-a)^{k}\right| \leq \delta(x-a)^{v-1},
$$

and

$$
\left|f(x)-\sum_{k=0}^{n-1} \frac{f^{(k)}(b)}{k !}(x-b)^{k}\right| \leq \delta(b-x)^{v-1},
$$

$\forall x \in[\mathrm{a}, \mathrm{b}]$.

As in the proof of Theorem 4, we get:

$$
\begin{gathered}
\left|\int_{a}^{b} f(x) d x-\sum_{k=0}^{n-1} \frac{1}{(k+1) !}\left[f^{(k)}(a)(t-a)^{k+1}+(-1)^{k} f^{(k)}(b)(b-t)^{k+1}\right]\right| \leq \\
\frac{\delta}{v}\left[(t-a)^{v}+(b-t)^{v}\right],
\end{gathered}
$$

$\forall \mathrm{t} \in[\mathrm{a}, \mathrm{b}]$.

The rest of the proof is similar to the proof of Theorem 4.

We continue with

Theorem 6. Let $\mathrm{p}, \mathrm{q}>1: \frac{1}{\mathrm{p}}+\frac{1}{\mathrm{q}}=1, v>\frac{1}{\mathrm{q}}, \mathrm{n}=\lceil\nu\rceil ; \mathrm{f} \in A C^{\mathrm{n}}([\mathrm{a}, \mathrm{b}])$, with $\mathrm{D}_{* \mathrm{a}}^{v} \mathrm{f}, \mathrm{D}_{\mathrm{b}-}^{v} \mathrm{f} \in$ $\mathrm{L}_{\mathrm{q}}([\mathrm{a}, \mathrm{b}])$. Then

i)

$$
\begin{gathered}
\left|\int_{a}^{b} f(x) d x-\sum_{k=0}^{n-1} \frac{1}{(k+1) !}\left[f^{(k)}(a)(t-a)^{k+1}+(-1)^{k} f^{(k)}(b)(b-t)^{k+1}\right]\right| \leq \\
\quad \frac{\max \left\{\left\|D_{* a}^{v} f\right\|_{L_{q}([a, b])},\left\|D_{b-}^{v} f\right\|_{L_{q}([a, b])}\right\}}{\Gamma(v)\left(v+\frac{1}{p}\right)(p(v-1)+1)^{\frac{1}{p}}}\left[(t-a)^{v+\frac{1}{p}}+(b-t)^{v+\frac{1}{p}}\right],
\end{gathered}
$$

$\forall \mathrm{t} \in[\mathrm{a}, \mathrm{b}]$,

ii) at $\mathrm{t}=\frac{\mathrm{a}+\mathrm{b}}{2}$, the right hand side of (52) is minimized, and we get:

$$
\left|\int_{a}^{b} f(x) d x-\sum_{k=0}^{n-1} \frac{1}{(k+1) !} \frac{(b-a)^{k+1}}{2^{k+1}}\left[f^{(k)}(a)+(-1)^{k} f^{(k)}(b)\right]\right| \leq
$$




$$
\frac{\max \left\{\left\|D_{* a}^{v} f\right\|_{L_{q}([a, b])},\left\|D_{b-}^{v} f\right\|_{L_{q}([a, b])}\right\}}{\Gamma(v)\left(v+\frac{1}{p}\right)(p(v-1)+1)^{\frac{1}{p}}} \frac{(b-a)^{v+\frac{1}{p}}}{2^{v-\frac{1}{q}}},
$$

iii) if $\mathrm{f}^{(\mathrm{k})}(\mathrm{a})=\mathrm{f}^{(\mathrm{k})}(\mathrm{b})=0$, for all $\mathrm{k}=0,1, \ldots, \mathrm{n}-1$, we obtain

$$
\left|\int_{a}^{b} f(x) d x\right| \leq \frac{\max \left\{\left\|D_{* a}^{v} f\right\|_{L_{q}([a, b])},\left\|D_{b-}^{v} f\right\|_{L_{q}([a, b])}\right\}}{\Gamma(v)\left(v+\frac{1}{p}\right)(p(v-1)+1)^{\frac{1}{p}}} \frac{(b-a)^{v+\frac{1}{p}}}{2^{v-\frac{1}{q}}},
$$

which is a sharp inequality,

iv) more generally, for $\mathbf{j}=0,1,2, \ldots, \mathbf{N} \in \mathbb{N}$, it holds

$$
\begin{aligned}
& \left|\int_{a}^{b} f(x) d x-\sum_{k=0}^{n-1} \frac{1}{(k+1) !}\left(\frac{b-a}{N}\right)^{k+1}\left[j^{k+1} f^{(k)}(a)+(-1)^{k}(N-j)^{k+1} f^{(k)}(b)\right]\right| \\
& \leq \frac{\max \left\{\left\|D_{* a}^{v} f\right\|_{L_{q}([a, b])},\left\|D_{b-}^{v} f\right\|_{L_{q}([a, b])}\right\}}{\Gamma(v)\left(v+\frac{1}{p}\right)(p(v-1)+1)^{\frac{1}{p}}}\left(\frac{b-a}{N}\right)^{v+\frac{1}{p}}\left[j^{v+\frac{1}{p}}+(N-j)^{v+\frac{1}{p}}\right],
\end{aligned}
$$

v) if $\mathrm{f}^{(\mathrm{k})}(\mathrm{a})=\mathrm{f}^{(\mathrm{k})}(\mathrm{b})=0, \mathrm{k}=1, \ldots, \mathrm{n}-1$, from (55) we get:

$$
\begin{gathered}
\left|\int_{a}^{b} f(x) d x-\left(\frac{b-a}{N}\right)[j f(a)+(N-j) f(b)]\right| \leq \\
\frac{\max \left\{\left\|D_{* a}^{v} f\right\|_{L_{q}([a, b])},\left\|D_{b-}^{v} f\right\|_{L_{q}([a, b])}\right\}}{\Gamma(v)\left(v+\frac{1}{p}\right)(p(v-1)+1)^{\frac{1}{p}}}\left(\frac{b-a}{N}\right)^{v+\frac{1}{p}}\left[j^{v+\frac{1}{p}}+(N-j)^{v+\frac{1}{p}}\right],
\end{gathered}
$$

for $\mathrm{j}=0,1,2, \ldots, \mathrm{N}$,

vi) when $\mathrm{N}=2$ and $\mathrm{j}=1$, (56) turns to

$$
\begin{gathered}
\left|\int_{a}^{b} f(x) d x-\left(\frac{b-a}{2}\right)(f(a)+f(b))\right| \leq \\
\frac{\max \left\{\left\|D_{* a}^{v} f\right\|_{L_{q}([a, b])},\left\|D_{b-}^{v} f\right\|_{L_{q}([a, b])}\right\}}{\Gamma(v)\left(v+\frac{1}{p}\right)(p(v-1)+1)^{\frac{1}{p}}} \frac{(b-a)^{v+\frac{1}{p}}}{2^{v-\frac{1}{q}}},
\end{gathered}
$$

vii) when $1 / \mathrm{q}<v \leq 1$, inequality (57) is again valid but without any boundary conditions.

Proof. Here $v>0, n=\lceil v\rceil, f \in A C^{n}([a, b]) ; p, q>1: \frac{1}{p}+\frac{1}{q}=1$, with $D_{* a}^{v} f, D_{b-}^{v} f \in L_{q}([a, b])$. By (10) we have

$$
\left|f(x)-\sum_{k=0}^{n-1} \frac{f^{(k)}(a)}{k !}(x-a)^{k}\right| \leq \frac{1}{\Gamma(v)} \int_{a}^{x}(x-t)^{v-1}\left|D_{* a}^{v} f(t)\right| d t \leq
$$




$$
\begin{gathered}
\frac{1}{\Gamma(v)}\left(\int_{a}^{x}(x-t)^{p(v-1)} d t\right)^{\frac{1}{p}}\left(\int_{a}^{x}\left|D_{* a}^{v} f(t)\right|^{q} d t\right)^{\frac{1}{q}} \leq \\
\frac{1}{\Gamma(v)} \frac{(x-a)^{\frac{p(v-1)+1}{p}}}{(p(v-1)+1)^{\frac{1}{p}}}\left\|D_{* a}^{v} f\right\|_{L_{q}([a, b])} .
\end{gathered}
$$

Here we assume that $v>\frac{1}{q} \Leftrightarrow p(v-1)+1>0$. So, we get

$$
\left|f(x)-\sum_{k=0}^{n-1} \frac{f^{(k)}(a)}{k !}(x-a)^{k}\right| \leq \frac{\left\|D_{* a}^{v} f\right\|_{L_{q}([a, b])}}{\Gamma(v)(p(v-1)+1)^{\frac{1}{p}}}(x-a)^{v-\frac{1}{q}},
$$

$\forall x \in[\mathrm{a}, \mathrm{b}]$.

By (11) we have

$$
\begin{gathered}
\left|f(x)-\sum_{k=0}^{n-1} \frac{f^{(k)}(b)}{k !}(x-b)^{k}\right| \leq \frac{1}{\Gamma(v)} \int_{x}^{b}(z-x)^{v-1}\left|D_{b-}^{v} f(z)\right| d z \leq \\
\frac{1}{\Gamma(v)}\left(\int_{x}^{b}(z-x)^{p(v-1)} d z\right)^{\frac{1}{p}}\left(\int_{x}^{b}\left|D_{b-}^{v} f(z)\right|^{q} d z\right)^{\frac{1}{q}} \leq \\
\frac{1}{\Gamma(v)} \frac{(b-x)^{\frac{p(v-1)+1}{p}}}{(p(v-1)+1)^{\frac{1}{p}}}\left\|D_{b-}^{v} f\right\|_{L_{q}([a, b])} .
\end{gathered}
$$

So, we get

$$
\left|f(x)-\sum_{k=0}^{n-1} \frac{f^{(k)}(b)}{k !}(x-b)^{k}\right| \leq \frac{\left\|D_{b-}^{v} f\right\|_{L_{q}([a, b])}}{\Gamma(v)(p(v-1)+1)^{\frac{1}{p}}}(b-x)^{v-\frac{1}{q}},
$$

$\forall x \in[\mathrm{a}, \mathrm{b}]$.

Call

$$
\rho_{1}:=\frac{\left\|D_{* a}^{v} f\right\|_{L_{q}([a, b])}}{\Gamma(v)(p(v-1)+1)^{\frac{1}{p}}}
$$

and

$$
\rho_{2}:=\frac{\left\|D_{b-}^{v} f\right\|_{L_{q}([a, b])}}{\Gamma(v)(p(v-1)+1)^{\frac{1}{p}}} .
$$

Set

$$
\rho:=\max \left(\rho_{1}, \rho_{2}\right), \quad m:=v-\frac{1}{q}>0 .
$$

That is

$$
\left|f(x)-\sum_{k=0}^{n-1} \frac{f^{(k)}(a)}{k !}(x-a)^{k}\right| \leq \rho(x-a)^{m},
$$

and

$$
\left|f(x)-\sum_{k=0}^{n-1} \frac{f^{(k)}(b)}{k !}(x-b)^{k}\right| \leq \rho(b-x)^{m},
$$


$\forall x \in[\mathrm{a}, \mathrm{b}]$.

As in the proof of Theorem 4, we obtain:

$$
\begin{gathered}
\left|\int_{a}^{b} f(x) d x-\sum_{k=0}^{n-1} \frac{1}{(k+1) !}\left[f^{(k)}(a)(t-a)^{k+1}+(-1)^{k} f^{(k)}(b)(b-t)^{k+1}\right]\right| \leq \\
\frac{\rho}{(m+1)}\left[(t-a)^{m+1}+(b-t)^{m+1}\right]= \\
\frac{\max \left\{\left\|D_{* a}^{v} f\right\|_{L_{q}([a, b])},\left\|D_{b-}^{v} f\right\|_{L_{q}([a, b])}\right\}}{\Gamma(v)(p(v-1)+1)^{\frac{1}{p}}\left(v+\frac{1}{p}\right)}\left[(t-a)^{v+\frac{1}{p}}+(b-t)^{v+\frac{1}{p}}\right],
\end{gathered}
$$

$\forall \mathrm{t} \in[\mathrm{a}, \mathrm{b}]$.

The rest of the proof is similar to the proof of Theorem 4.

\section{References}

[1] George A. Anastassiou, Fractional Differentiation Inequalities, Springer, Heidelberg, New York, 2009.

[2] George A. Anastassiou, Intelligent Mathematical Computational Analysis, Springer, Heidelberg, New York, 2011.

[3] K. Diethelm, The Analysis of Fractional Differential Equations, Springer, Heidelberg, New York, 2010.

[4] K.S.K. Iyengar, Note on an inequality, Math. Student, 6 (1938), 75-76. 